\title{
Assessing the effects of Kampo medicine on human skin texture and microcirculation
}

\author{
Hongyang $\mathrm{Li}^{1,2} \cdot$ Tomokazu Yoshizaki $^{1} \cdot$ Lian Liang $^{2} \cdot$ Mako Iwahashi $^{2} \cdot$ Akihiro Kawahara $^{3} \cdot$ Akiko Shirai $^{1}$. \\ Junsuke Arimitsu ${ }^{2} \cdot$ Masanori Ito $^{3} \cdot$ Norimichi Tsumura $^{4} \cdot K_{\text {Keiko Ogawa-Ochiai }}^{2}$
}

Received: 8 November 2021 / Accepted: 4 January 2022 / Published online: 24 January 2022

(c) International Society of Artificial Life and Robotics (ISAROB) 2022

\begin{abstract}
In this study, we verified the effectiveness of Kampo medicine by evaluating the changes in the feature values of facial skin texture and microcirculation at two distinct tissue depths (subcutaneous $2 \mathrm{~mm}$ and $8 \mathrm{~mm}$ ). A total of 80 patients who took the Kampo formula participated in this study, and the changes in the feature values of facial skin texture and microcirculation were measured before and after Kampo treatment. The treatment period lasted 6-18 months, according to the doctor's judgment. The total area of the sulci cutis and the average thickness of the sulci cutis significantly decreased $(P<0.05)$, and the pixels of the grayscale image increased after Kampo treatment $(P<0.05)$. Moreover, the blood flow velocity at $8 \mathrm{~mm}$ depth significantly increased after Kampo treatment $(P<0.05)$. In this study, we specifically noted changes in the skin texture and microcirculation after Kampo treatment.
\end{abstract}

Keywords Kampo medicine $\cdot$ Microcirculation $\cdot$ Skin texture $\cdot$ Inspection

\section{Introduction}

Kampo medicine (traditional Japanese medicine) is a medical system that is rooted in ancient Chinese medicine, which has been systematically adapted to suit the climate, environment, and culture of Japan. The diagnostic approach applied in Kampo medicine has become popular worldwide and the treatment has achieved excellent outcomes for several diseases [1, 2]. Recently, coronavirus disease 2019 (COVID19) has been the center of attention, and many studies have

Keiko Ogawa-Ochiai

ikkandoo@gmail.com

1 Department of Otolaryngology-Head and Neck Surgery, Kanazawa University Hospital, 13-1 Takaramachi, Kanazawa-city, Ishikawa 920-8641, Japan

2 Department of General Internal Medicine, Kampo Clinical Center, Hiroshima University Hospital, 1-2-3 Kasumi Hiroshima-city, Hiroshima 734-8551, Japan

3 Department of General Internal Medicine, Hiroshima University Hospital, 1-2-3 Kasumi Hiroshima-city, Hiroshima 734-8551, Japan

4 Graduate School of Engineering, Chiba University, 1-33, Yayoi-cho, Inage-ku, Chiba-shi, Chiba 263-8522, Japan shown that Kampo medicine can reduce the incidence rate and severity of cases [3, 4].

In Kampo medicine, there are four diagnostic methods: inspection, listening and smelling examination, inquiry, and palpation. Inspection includes a visual examination of the complexion, tongue, and secretions. The Yellow Emperor's Eighty-one Difficulties Classic noted the following: "That who can know the pathogenesis of the disease by observing the patient's complexion, he can be called a god." Thus, examination of the complexion is the most important part of the inspection. During the inspection process, doctors diagnose diseases visually, according to their subjective judgment and personal knowledge. Therefore, it is difficult for patients and medical interns to understand the process. Thus, it would be beneficial to objectify the process of inspection.

Recently, considerable research has been conducted to assess skin texture in the cosmetic industry. For instance, Takemae et al. built an automated system for the evaluation of human skin surface conditions using image processing [5], and Kobayashi et al. also built a system to assess skin texture using image processing [6]. However, while they obtained skin images using a contact method, Uchida et al. improved the skin texture evaluation systems by using a noncontact method [7]. Kawasaki et al. showed that Uchida's 
non-contact skin texture evaluation method can be used for the inspection process in Kampo medicine [8].

Qi (vital energy) and blood are two of the vital substances in Kampo medicine. Therefore, it is also necessary to measure the oxygen saturation and blood flow in patients. This is especially true for patients with qi or blood deficiency. O2C ("Oxygen-to-see," LEA Medizintechnik, Giessen, Germany) is a new diagnostic device for the noninvasive determination of tissue oxygenation and microcirculation [9]. The measurement principle of the device is a combination of the laser Doppler technique and tissue spectrometry. In the present study, a flat, glass fiber probe was connected to the $\mathrm{O} 2 \mathrm{C}$ device to measure microcirculation parameters (venous oxygen saturation $\left[\mathrm{SvO}_{2}\right]$, hemoglobin concentration [rHb], blood flow [flow], and flow velocity [velocity]) at two depths: $2 \mathrm{~mm}$ (cutaneous tissue) and $8 \mathrm{~mm}$ (subcutaneous tissue). As Fig. 1 shows the cutaneous tissue is full of Capillary vessels, and the subcutaneous tissue is full of large vessels. The device is used in many conditions, such as wound healing [10], plastic surgery [11], and diabetes (diabetic foot/arterial occlusive diseases) [12]. This is the first time it has been used in the field of Kampo medicine.

The aim of this study was to verify the effectiveness of the Kampo medicine treatment by evaluating the changes in the feature values of facial skin texture and microcirculation, and to develop the concept of inspection.

\section{Methods}

\subsection{Subjects}

This was a prospective observational study [13]. Eighty patients (mean age: $51.58 \pm 16.35$ years) who took Kampo formulae participated in this study. The doctor of Kampo medicine prescribed the Kampo formula for the patients

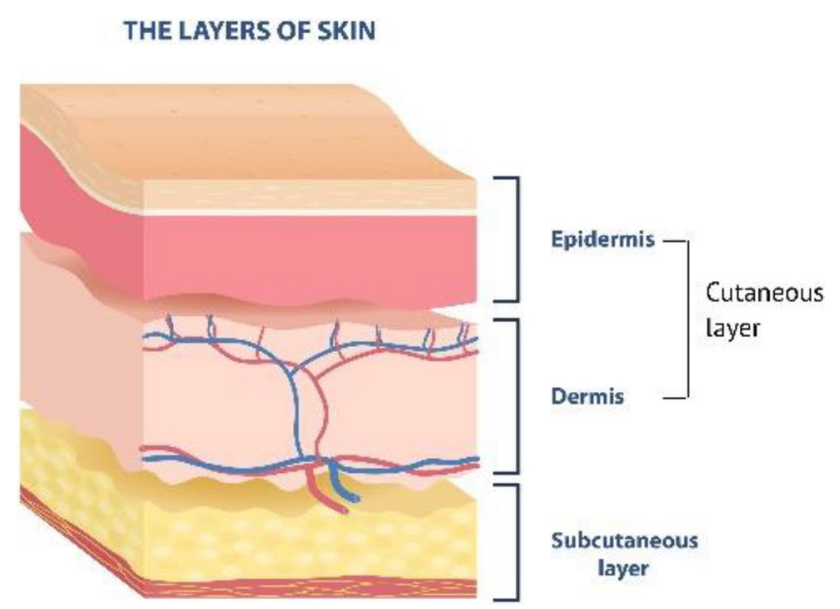

Fig. 1 Layers of skin according to their diagnosis. The treatment period lasted from six to eighteen months, at the doctor's discretion.

The study was approved by the Medical Ethics Committee of Kanazawa University, approval no. 2018-154 (2912). The study commenced in April 2019 and terminated in February 2021.

\subsection{Outcome measurement}

The outcome was the change in feature values of the facial skin texture and microcirculation at two distinct tissue depths (2 $\mathrm{mm}$ and $8 \mathrm{~mm}$ subcutaneous).

\subsubsection{Feature values of the facial skin texture}

We captured images of the facial skin (the left cheek) using a digital camera (NIKON D5100), lens (TAMRON Model F017) and an LED fill light with a transparent filter (NEEWER 176 LED VIDEO LIGHT) before and after Kampo treatment. Figure 2 shows the experimental setup. The distance between the patient and the camera was $0.5 \mathrm{~m}$. The F number of the lens was 36 , the shutter speed was $1 / 4$, the ISO speed was 200, and the image size was set to large pixels. To prevent blurring, shutter release was used. The brightness of the LED lights was $100 \%$, and the color temperature was $5900 \mathrm{~K}$. The images were taken in the same room, the room temperature was set to $24-26{ }^{\circ} \mathrm{C}$, and humidity was 45-60\%.

We converted the original image format to TIF using Nikon ViewNX 2 software, and then used the Microsoft Paint software to trim the images, to make sure the trimming are taken in the same location, the images of patients before and after treatment were processed simultaneously, and the same facial markers were selected, the size of trimmed images was $701 \times 701$ pixels. We analyzed images using the non-contact skin texture evaluation method

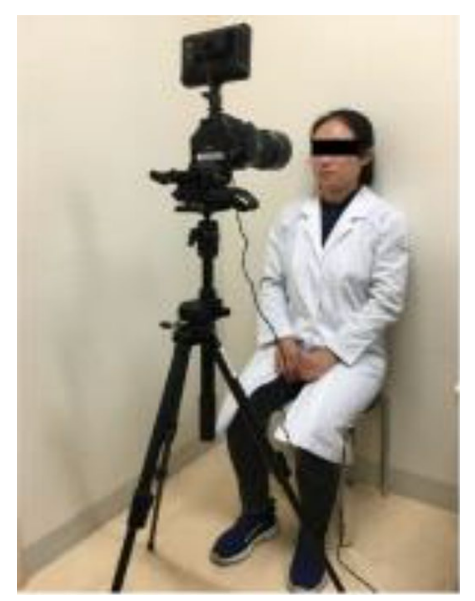

Fig. 2 The experimental setting 
(Fig. 3) using MATLAB R2018a, as used at Chiba University [according to the flow: (1) Grayscale conversion (2) Cross-binarization (3) Thinning (4) Short-line matching] [7]. The feature values analyzed from facial images included the average thickness of the sulci cutis, total area of the sulci cutis, standard deviation (SD) of short-line inclination angles, and pixels of the grayscale image.

\subsubsection{The detection of microcirculation}

The $\mathrm{O} 2 \mathrm{C}$ probe (flat type) gently adhered to the middle of the dorsal aspect of the right forearm, $5 \mathrm{~cm}$ above the transverse crease of the wrist (avoiding large vessels) with double-sided, transparent, adhesive tape, as shown in Fig. 4. The depth resolution of the device is $1 \mathrm{~nm}$. Measurement of microcirculation was initiated after the patient was sitting for $10 \mathrm{~min}$. The patients were seated in an upright position with their hands on their knees, and measurement continued for $3 \mathrm{~min}$, during the measurement we asked the patients to stay still and count numbers in heads. Microcirculation was assessed before and after Kampo treatment. Venous oxygen saturation, hemoglobin concentration, blood flow, and flow velocity were obtained at tissue depths of $2 \mathrm{~mm}$ and $8 \mathrm{~mm}$. The data is an average value of $3 \mathrm{~min}$. The room was set to a comfortable temperature $\left(24-26^{\circ} \mathrm{C}\right)$ for each patient.

\subsection{Statistical analysis}

Statistical analyses were performed using SPSS software (IBM SPSS Statistics ver. 19). The Kolmogorov-Smirnov test was used to test for normal distribution of the values. The changes in feature values and microcirculation before

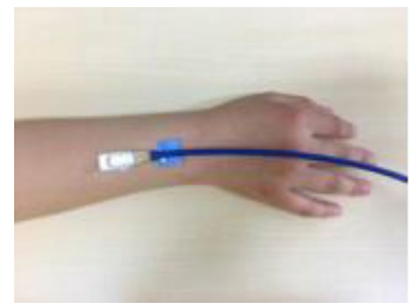

Fig. 4 Probe position

and after the treatment were compared using the paired sample $t$-test or Wilcoxon signed-rank test. The level of significance was set at $P<0.05$.

\section{Results}

A total of 80 patients ( 21 men and 59 women) participated in this study. The prescriptions and treatment durations are shown in Table 1. Some Kampo medicine prescriptions were used in combination.

The changes in the feature values are shown in Fig. 5, 6, and 7 and Table 2. The total area of the sulci cutis significantly decreased after Kampo treatment $(P<0.05)$. The average thickness of the sulci cutis also significantly decreased after Kampo treatment $(P<0.05)$. The pixels in the grayscale image significantly increased after Kampo treatment $(P<0.05)$. However, there were no significant differences in the SD of the short-line inclination angles $(P>0.05)$.

The changes in the microcirculation are shown in Fig. 8 and Table 3 . Only blood flow velocity at a depth of $8 \mathrm{~mm}$

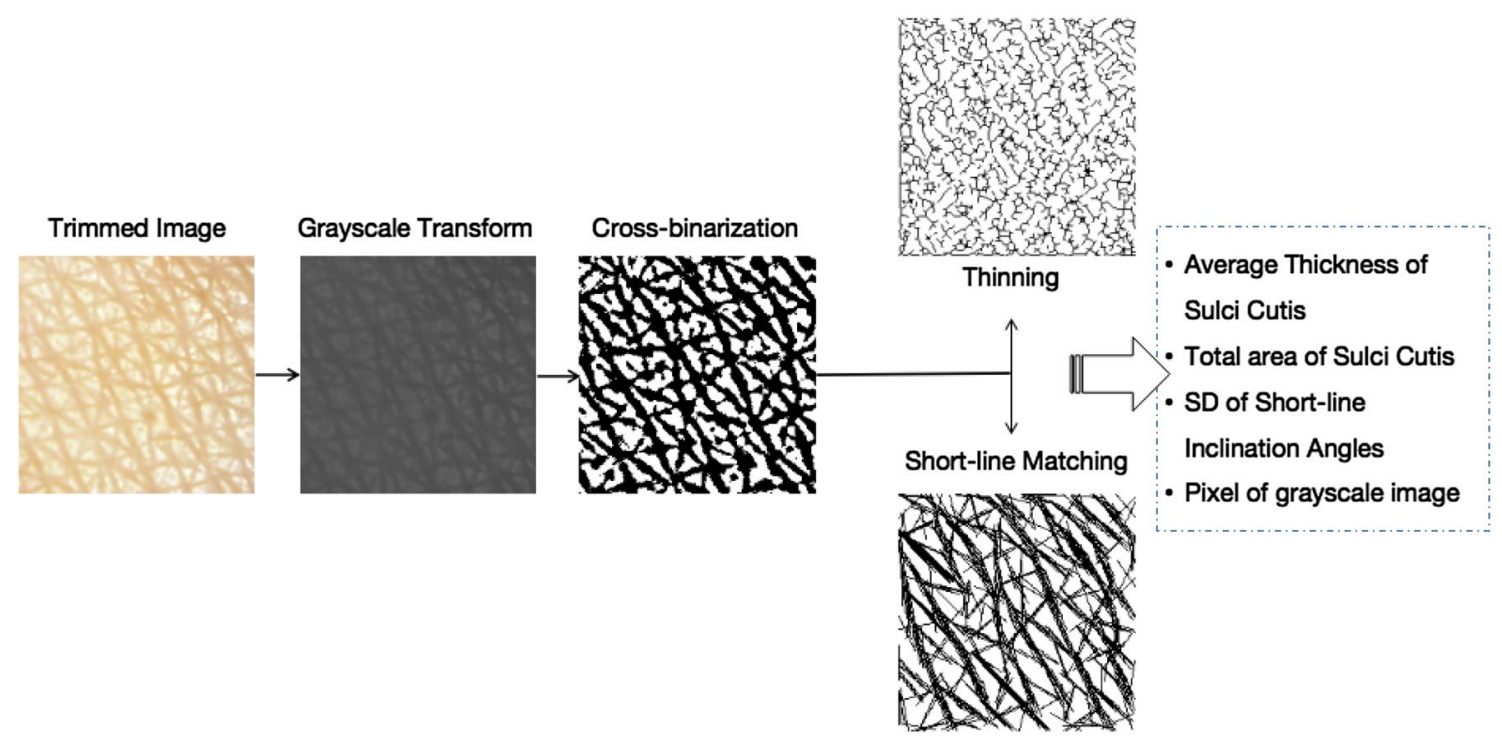

Fig. 3 The skin texture feature extraction technology 
Table 1 Patient demographic characteristics

\begin{tabular}{lll}
\hline & $n$ & Mean \pm SD \\
\hline Gender and age & 21 & \\
Male & 59 & $54.71 \pm 18.13$ \\
Female & 80 & $51.58 \pm 16.35$ \\
Total & & \\
Prescription & 9 & \\
Tokishakuyakusan & 13 & \\
Ninjinyoeito & 9 & \\
Goreisan & 17 & \\
Hatimigann & 8 & \\
Shitimotukoukatou & & \\
Other & & \\
Treatment duration & 19 & \\
0.5 years & 32 & \\
$0.5-1$ year & 29 & \\
1-1.5 years & & \\
\hline
\end{tabular}

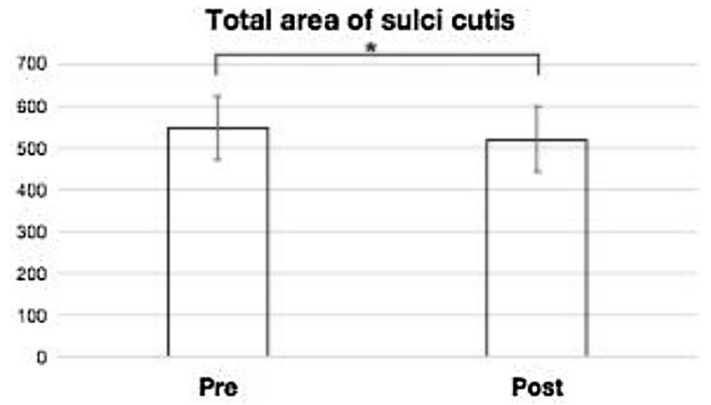

Fig. 5 Change in the total area of the sulci cutis before and after the Kampo treatment. $* P<0.05$

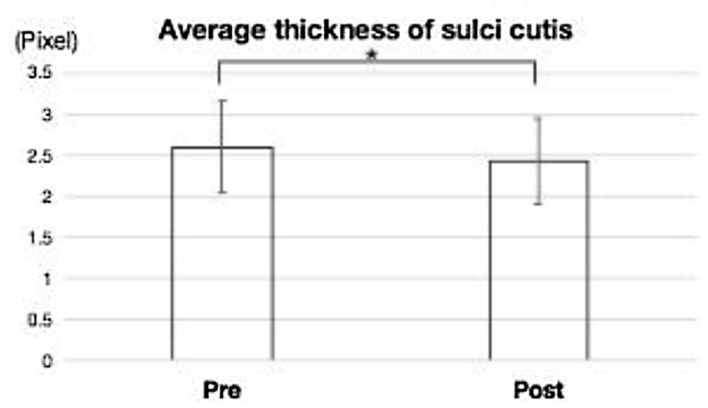

Fig. 6 Change in the average thickness of the sulci cutis before and after the Kampo treatment. $* P<0.05$

significantly increased after Kampo treatment $(P<0.05)$. There was no significant difference in hemoglobin oxygenation, hemoglobin concentration, and blood flow $(P>0.05)$.

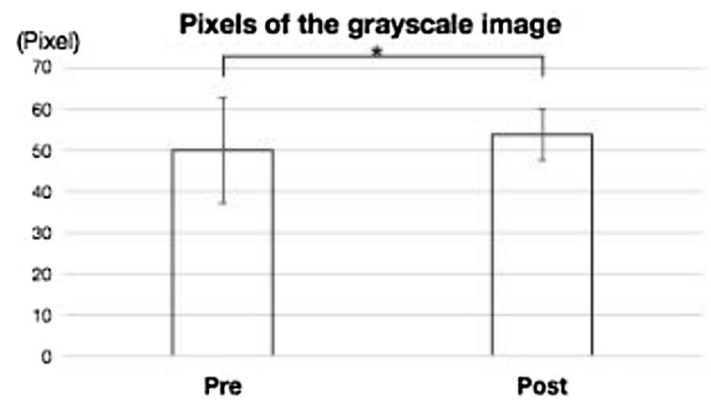

Fig. 7 Change in the pixel number of the grayscale image before and after the Kampo treatment. $* P<0.05$

\section{Discussion}

In this study, we found that blood flow velocity at a depth of $8 \mathrm{~mm}$ increased after patients received a Kampo formula. Numerous studies have also verified that Kampo medicine can promote blood flow. Ge et al. found that traditional Chinese medicine can effectively improve microcirculation in arteriosclerotic obliteration (ASO) patients and accelerate blood flow in the lower limbs [14]. Zhang et al. also found that the Shexiang Tongxin dropping pill improves peripheral microvascular blood flow [15]. These two studies indicate similar changes in microcirculation as were found in our study. In Kampo medicine, qi and blood are two of the vital substances, so in Kampo treatment, doctors always attach great importance to qi and blood. Qi is the vitality that circulates through the body day and night [16], and blood and qi have a very close relationship. Qi can generate blood, move blood, and hold blood [17], so it is said that qi is the leader of blood: if qi moves, blood follows, but if qi stagnates, blood coagulates. At the same time, blood can nourish qi [17], and it is said that blood is the mother of qi. Most Kampo formulae contain qi and blood tonifying drugs. Therefore, in this study, we believe that the Kampo formulae accelerated blood flow by regulating the qi and blood of patients.

In this study, we did not see a significant change of microcirculation at $2 \mathrm{~mm}$. The microcirculation at a depth of $2 \mathrm{~mm}$ represents cutaneous tissue, whereas a depth of $8 \mathrm{~mm}$ represents subcutaneous tissue [18]. Compared with subcutaneous tissue, cutaneous tissue is more easily affected by the external environment, this could explain why no changes in microcirculation in response to Kampo treatment were found at $2 \mathrm{~mm}$.

Studies on Kampo treatment and changes in facial skin texture are rare. In this study, we found that the total area and average thickness of the sulci cutis decreased after Kampo treatment. The skin surface has irregular sulcus cutis and crista cutis. The sulcus cutis describes grooves and depressions in the skin, and the areas surrounded by 
Table 2 Changes in feature values of the facial skin texture

\begin{tabular}{llllr}
\hline & Total area & $\begin{array}{l}\text { Average thickness } \\
\text { (pixel) }\end{array}$ & SD of short-line & Pixel (pixel) \\
\hline Pre-treatment & $544.45 \pm 76.00$ & $2.59 \pm 0.56$ & $30.22 \pm 6.81$ & $49.69 \pm 12.80$ \\
Post-treatment & $518.99 \pm 79.01^{*}$ & $2.42 \pm 0.53^{*}$ & $31.84 \pm 6.49$ & $53.63 \pm 6.11^{*}$ \\
\hline
\end{tabular}

$* P<0.05$, compared with pre-treatment

Total area: The total area of the sulci cutis

Average thickness: The average thickness of the sulci cutis

SD of short-line: The SD of the short-line inclination angles (expresses the variety of directions of the sulci cutis)

Pixel: The pixels in the grayscale image

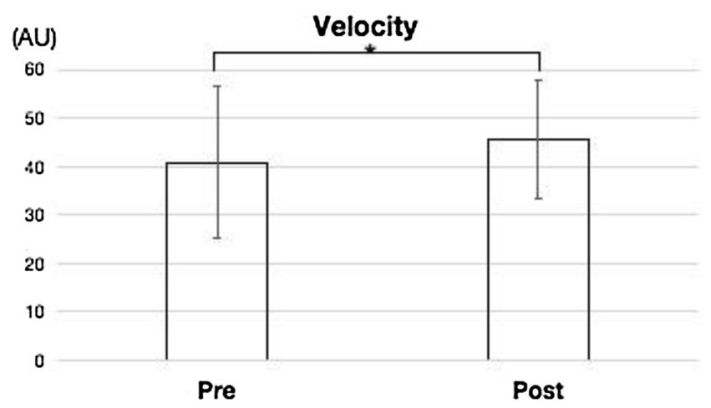

Fig. 8 Change in the blood flow velocity before and after the Kampo treatment. $* P<0.05$

Table 3 Changes in microcirculation parameters

\begin{tabular}{llll}
\hline & & Pre-treatment & Post-treatment \\
\hline $2 \mathrm{~mm}$ & $\mathrm{SvO}_{2}(\%)$ & $38.00 \pm 11.49$ & $38.69 \pm 12.50$ \\
& $\mathrm{rHb}(\mathrm{AU})$ & $57.70 \pm 8.27$ & $57.28 \pm 8.64$ \\
& Flow (AU) & $13.07 \pm 16.65$ & $10.15 \pm 10.43$ \\
& Velocity (AU) & $29.35 \pm 7.74$ & $29.97 \pm 8.52$ \\
$8 \mathrm{~mm}$ & $\mathrm{SvO}_{2}(\%)$ & $32.79 \pm 18.50$ & $29.97 \pm 17.57$ \\
& $\mathrm{rHb}(\mathrm{AU})$ & $67.79 \pm 18.97$ & $65.23 \pm 18.94$ \\
& Flow (AU) & $32.71 \pm 34.34$ & $31.43 \pm 20.67$ \\
& Velocity (AU) & $40.84 \pm 15.76$ & $45.64 \pm 12.25 *$ \\
\hline
\end{tabular}

$* P<0.05$, compared with pre-treatment

$A U$ arbitrary unit

it are called crista cutis [19] (Fig. 9). Given that the area of the sulci cutis can be influenced by the quantity of sulci cutis and the thickness of sulci cutis, we suggest two probable causes for the reduction of the area of the sulci cutis: a reduction in the number and thickness of sulci cutis. In this study, we verified that sulci cutis thickness decreased after Kampo treatment. However, we did not quantify the number of sulci cutis. In agreement with these findings, Nitta et al. also verified that the average thickness of sulci

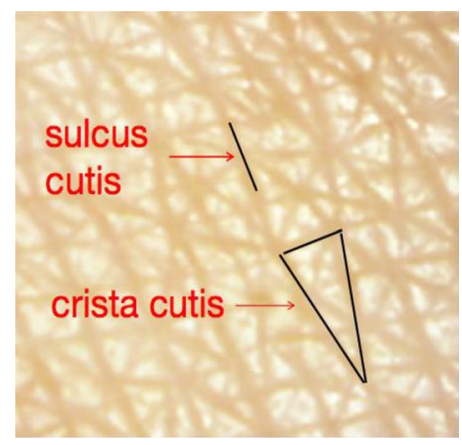

Fig. 9 Skin texture

cutis significantly decreased after dry-skin care in older adults, and found that when water levels in the stratum corneum increased, the stratum corneum became tight and the volume of cristae cutis were increased, thus narrowing sulci cutis between cristae cutis [20]. As skin ages, it loses its ability to retain moisture, so most elderly people suffer from rough and dry skin [21]. The majority of the patients in our study were elderly. Many studies have shown that Kampo medicine can moisturize the skin by replenishing qi and blood. For example, Takada et al. found that the water absorbing and holding capacities of the skin were improved by ingesting Shimotsu-to [22], and Nakamura et al. found that Ninjinyoei-to is useful for dry skin [23].

In this study, we also found that the pixels of the grayscale images increased after Kampo treatment. The value range of the pixels of the grayscale images is 0 pixel to 255 pixels, 0 pixel expresses dark and 255 pixels expresses bright, so the increasing of the pixels of the grayscale images indicated that the facial skin became brighter. Numerous studies verified that skin brightness increased as the skin became moisturized [24, 25]. Therefore we suggest that Kampo medicine can reduce the average thickness of the sulci cutis and increase skin brightness by improving skin moisture content. 


\section{Limitations and future work}

The limitations of this study include that we focused on skin texture and did not assess other parameters of the skin, such as temperature and moisture. Additionally, because of the small sample size we did not assess the changes associated with different ailments or Kampo formulae separately, future work with a larger sample size is warranted to determine which patients experience microcirculation and skin texture changes after treatment.

\section{Conclusion}

In this study, we found that after Kampo treatment changes occurred in the skin texture and microcirculation. Facial skin texture can be measured objectively using image analysis technology.

Acknowledgements We thank Sakura Matsumoto and Ryouta Kiyomatsu for helping complete this study. This work is supported by the Nippon Foundation.

\section{References}

1. Kimata Y, Ogawa K, Okamoto H, Chino A, Namiki T (2016) Efficacy of Japanese traditional (Kampo) medicine for treating chemotherapy-induced peripheral neuropathy: a retrospective case series study. World J Clin Cases 4(10):310-317

2. Makita H, Endo K, Shirai A, Kawasaki K, Mishima R, Kasahara Y, Ishikawa K, Ueno T, Nakanishi Y, Kondo S, Wakisaka N, Yoshizaki T, Ogawa-Ochiai K (2020) Eppikajutsuto (a Japanese herbal medicine) regulates mTOR and induces apoptosis in oral cancer cells. Trad Kampo Med 7(2):72-77

3. Takayama S, Namiki T, Odaguchi H, Arita R, Hisanaga A, Mitani K (2021) Ito T (2021) Prevention and recovery of COVID-19 patients with Kampo medicine: review of case reports and ongoing clinical trials. Front Pharmacol 12:656246

4. Zheng S, Barak JP, Li S, Xiao W, Ren H, Yang H, Gan Y, Wen C (2020) Network pharmacology analysis of the therapeutic mechanisms of the traditional chinese herbal formula Lian Hua Qing Wen in corona virus disease 2019 (COVID-19), gives fundamental support to the clinical use of LHQW. Phytomedicine. https:// doi.org/10.1016/j.phymed.2020.153336

5. Takemae Y, Saito H, Ozawa S (2001) The evaluating system of human skin surface condition by image processing (In Japanese). Trans Soc Instrum Control Eng 37(11):1097-1103

6. Kobayashi H, Hashimoto T, Yamazaki K, Hirai Y (2010) Proposal of quantitative index of skin texture by the image processing and its practical application (In Japanese). Trans Jpn Soc Mech Eng Part C 76(764):922-929

7. Uchida M, Akaho R, Ogawa-Ochiai K, Tsumura N (2019) Imagebased measurement of changes to skin texture using piloerection for emotion estimation. Artif Life Robot 24(1):12-18

8. Kawasaki K, Li H, Mako Iwahashi M, Tsuda M, Takebe T, Shirai A, Tsumura N, Ogawa-Ochiai K (2020) Image-based non-contact monitoring of changes in facial skin texture induced by the contact needle technique of acupuncture. Artif Life Robot 25:356-362

9. Knobloch K, Kraemer R, Lichtenberg A, Jagodzinski M, Gosling T, Richter M, Krettek C (2006) Microcirculation of the ankle after Cryo/Cuff application in healthy volunteers. Int J Sports Med 27:250-255

10. Beckert S, Witte MB, Königsrainer A, Coerper S (2004) The impact of $\mathrm{O} 2 \mathrm{C}$ for the quantification of tissue ischemia in diabetic foot ulcers. Diabetes Care 27(12):2863-2867

11. Hölzle F, Rau A, Loeffelbein DJ, Mücke T, Kesting MR, Wolff KD (2010) Results of monitoring fasciocutaneous, myocutaneous, osteocutaneous and perforator flaps: 4-year experience with 166 cases. Int Oral Maxillofac Surg 39:21-28

12. Harrison DK, McCollum PT, Newton DJ, Hickman P, Jain AS (1994) Amputation level assessment using lightguide spectrophotometry. Prosthet Orthot Int 19:139-147

13. Song JW, Chung KC (2010) Observational studies: cohort and case-control studies. Plast Reconstr Surg 126(6):2234-2242

14. Ge X, Ge P, Peng Y, Chen Y (2003) The effects of Chinese medicines on microcirculation of nail folds and blood flow velocity of limbs in the patients with arteriosclerotic obliteration. J Tradit Chin Med 23(2):87-89

15. Zhang Y, Zhao J, He Z, Shi S, Liang C, Wu Z (2019) Shexiang Tongxin dropping pill improves peripheral microvascular blood flow via cystathionine- $\gamma$-Lyase. Med Sci Monit 25:6313-6321

16. Hüsch T, Tsaur I, Reiter M, Mager R, Haferkamp A (2014) Traditional Chinese medicine in urology. Der Urol Ausg A 53(11):1625-1632

17. Maciocia G (2015) The foundations of Chinese medicine: a comprehensive text. Churchill Livingstone, London, p 65

18. Sommer B, Berschin G, Sommer HM (2013) Microcirculation under an elastic bandage during rest and exercise-preliminary experience with the laser-Doppler spectrophotometry system O2C. J Sports Sci Med 12:414-421

19. Tse C, Shibata C, Takahashi H (2016) Skin analysis using sulcus cutic extraction. Comput Graph Vis Inform 163(7):1-6

20. Nitta S, Matsumoto M, Sugama J et al (2016) New quantitative indicators of evaluating the skin care regimen for older adults with dry skin by using the digital image analysis. J Nurs Sci Eng 3(2):93-100

21. Haroun MT (2003) Dry skin in the elderly. Geriatr Aging 6(6):41-44

22. Takada H, Nagai H, Okuma K et al (1987) Influence of Shimotsuto, Kampo medicine mainly composed of rehmanniae radix, on skin moisture holding capacity in patients with dermatosis desicans (In Japanese). Skin Res 29(4):774-782

23. Nakamura G (2020) Effect of Ninjinyoei-to on women's skin problems (In Japanese). Phila Kampo 80:16-18

24. Jiang ZX, DeLaCruz J (2011) Appearance benefits of skin moisturization. Skin Res Technol 17(1):51-55

25. Bianchini JM, Zhang Q, Hanna G, Flach CR, Wang H, Southall MD, Mendelsohn R, Randhawa M (2019) A unique gel matrix moisturizer delivers deep hydration resulting in significant clinical improvement in radiance and texture. Clin Cosmet Investig Dermatol 12:229-239

Publisher's Note Springer Nature remains neutral with regard to jurisdictional claims in published maps and institutional affiliations. 